\title{
New spectroscopic components in multiple systems. IV.`
}

\author{
A. A. Tokovinin ${ }^{1,2}$ and N. A. Gorynya ${ }^{3}$ \\ 1 Cerro Tololo Inter-American Observatory, Casilla 603, La Serena, Chile \\ 2 Sternberg Astronomical Institute, 13 Universitetsky prosp., 119899 Moscow, Russia \\ 3 Institute of Astronomy of Russian Acad. Sci., 48 Pyatnitskaya Str, 109017 Moscow, Russia \\ e-mail: gorynya@sai.msu.ru
}

Received 11 April 2001 / Accepted 15 May 2001

\begin{abstract}
New close sub-systems are reported and studied in eight visual multiple systems. Elements of one double-lined and eight single-lined spectroscopic orbits are given. Magnitudes, colors, spectral types and masses of individual components are estimated by combining all available data. The visual secondary component of the young binary HD 27638 is a double-lined pair of G-type dwarfs on a 17-day orbit with an unseen but massive $\left(>1.2 M_{\odot}\right)$ spectroscopic tertiary on an 8-year orbit. The invisible secondary of HD 28271A is also massive, but it is not seen, probably because of a high luminosity of the primary - an F7 star with fast axial rotation and an active chromosphere which evolves off the Main Sequence. Two additional companions are found in the visual triple system HD 179484.
\end{abstract}

Key words. binaries: spectroscopic - binaries: visual

\section{Introduction}

This work continues the publications of spectroscopic orbits for the components of late-type multiple stars observed with CORAVEL-type radial velocity spectrometer. In our first paper (Tokovinin 1997a, Paper I) the motivation for this observing program and the methods of data analysis are described. Subsequent orbits were published in Tokovinin (1998, Paper II) and in Tokovinin (1999a, Paper III). Here we present a further nine orbits, following the same strategy, which need not be repeated. The results of this study are incorporated into the current version of the Multiple Star Catalogue (Tokovinin 1997b).

The observing program on multiple stars is now almost finished. In this last paper, we give the orbital elements that could not be determined earlier because of some problems (long periods, high eccentricity, close or additional companions etc.). Naturally, most of these orbits are of lower quality than those published previously in this series, and some may qualify only as preliminary.

A correlation the Radial Velocity Meter (RVM) (Tokovinin 1987) was used for the measurements. Most observations which form the basis of this work were made

Send offprint requests to: A. A. Tokovinin, e-mail: atokovinin@ctio.noao.edu

* Table 4 is only available in electronic form at the CDS via anonymous ftp to cdsarc.u-strasbg.fr (130.79.128.5) or via

http://cdsweb.u-strasbg.fr/cgi-bin/qcat?J/A+A/374/227
Table 1. Object identification.

\begin{tabular}{lllll}
\hline \hline IDS(1900) & ADS & HD & HIP & Other \\
\hline $00010+5752$ & 61 & 123 & 518 & HR 5 \\
$03317+0015$ & 2644 & $22468 B$ & 16836 & HR 1099 \\
$04165+2523$ & 3161 & $27638 B$ & 20430 & HR 1369 \\
$04226+3008$ & 3243 & 28271 & $20904 / 07$ & HR 1406 \\
$04302+0957$ & 3317 & $29140 \mathrm{~B}$ & 21402 & HR 1458 \\
$15333-0827$ & 9728 & 139461 & $76603 / 02$ & HR 5816 \\
$18214+5135$ & 11328 & 169816 & 90141 & BD +51 2372 \\
$19077+3837$ & 12145 & 179484 & 94252 & BD +38 3466 \\
\hline \hline
\end{tabular}

in 1994-2000 with the 70-cm telescope located on the Moscow University campus and with the 1-m telescope of the Simeis Observatory in Crimea. The velocity zero point was determined by observations of several IAU velocity standards each night. The precision of radial velocities ranges from $0.3 \mathrm{~km} \mathrm{~s}^{-1}$ for bright stars to $1-2 \mathrm{~km} \mathrm{~s}^{-1}$ for faint objects or for low-contrast dips. Three observations were also made by AAT in 1994 with the CORAVEL spectrometer (Baranne et al. 1979) at the Haute Provence Observatory.

\section{Basic data}

Table 1 contains the identifications for the eight systems: IDS (1900) index, ADS number (Aitken 1932), HD number, Hipparcos number (ESA 1997), and other identifiers for the primary components. The ADS and HD numbers 


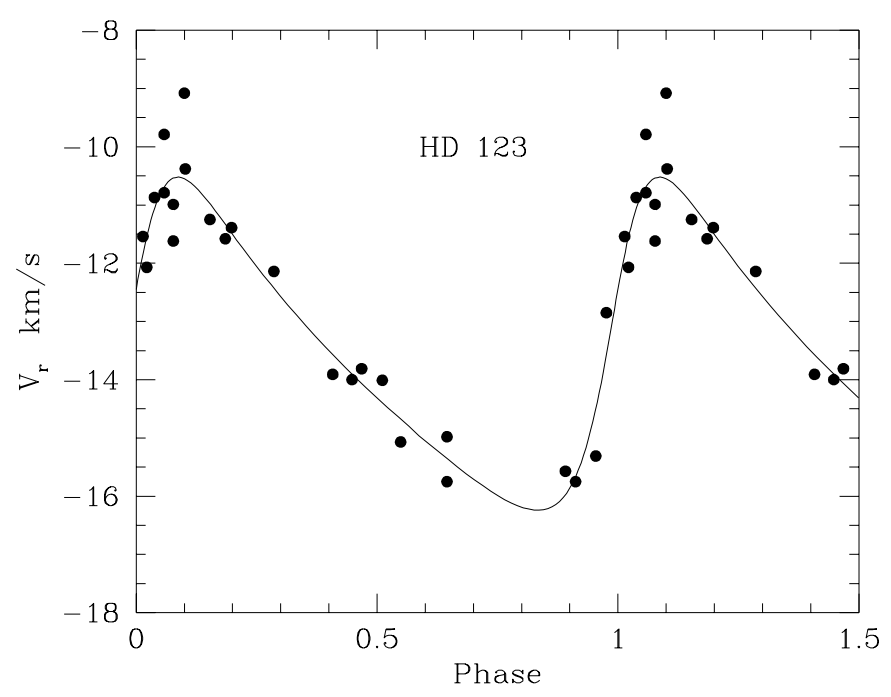

Fig. 1. Radial velocity curve of ADS $61 B=$ HD 123. The amplitude is reduced owing to the blending of the A and B dips.

exist for all these systems and they will be used throughout this paper.

Basic data on system components (spectral types, visual magnitudes and $B-V$ colors) are given in the left columns of Table 2 and were collected from the literature or from SIMBAD. Most of the photometry is taken from the Tycho catalogue (ESA 1997). Proper motions and trigonometric parallaxes are taken either from the Hipparcos catalogue or from other sources found through SIMBAD (in parentheses). The last four columns of Table 2 summarize the results of our study and contain the mean radial velocities, the number of measurements, the mean equivalent width $E W$ of the cross-correlation (CC) dip, and the projected axial rotational velocities $V \sin i$, as found from the width of the $\mathrm{CC}$ dip. The method of $V \sin i$ determination and the dependence of $E W$ on $B-V$ color and metallicity are described in Paper I and in Tokovinin (1990). Parameters of the CC dip are given separately for both components of the double-lined system HD 27638Bab.

\section{Spectroscopic orbits and system parameters}

The final orbital elements were found by least-squares fitting with weights inversely proportional to the square of formal velocity errors. The elements and their formal errors are given in Table 3 . The number of measurements used (primary and secondary lines counted separately) and the rms error of unit weight are given in the 9 th column of Table 3. Its last column contains mass function for single-lined systems or $M \sin ^{3} i$ for the components of the double-lined system. Individual observations, their errors and residuals can be found in Table 4 which is available in electronic form only. It contains also the measurements of non-variable components (a total of 403 velocities of fifteen components). The three CORAVEL measurements are marked as COR in the last column. The two

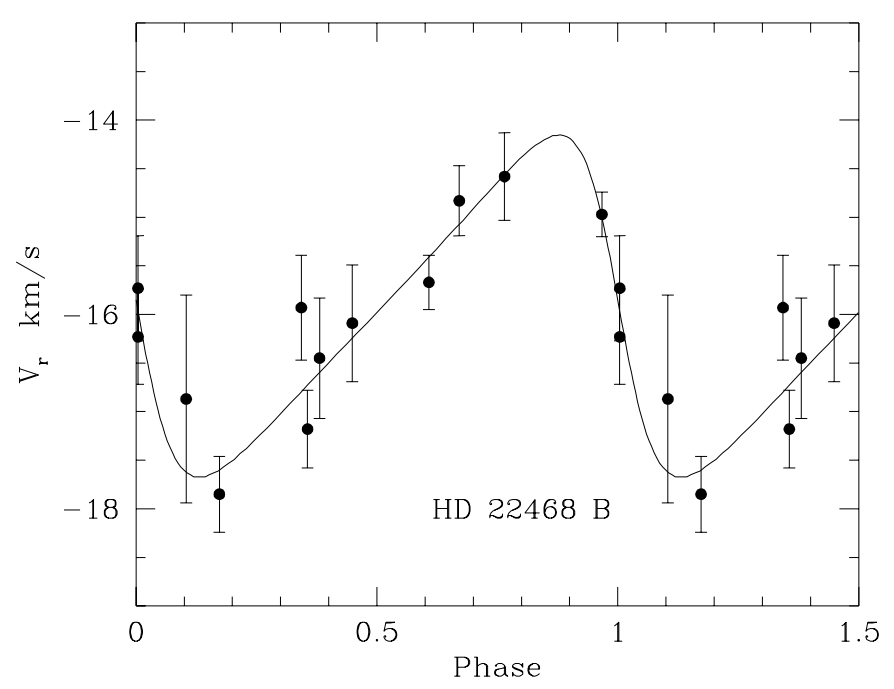

Fig. 2. Radial velocity curve of ADS 2644B = HD 22468B.

measurements rejected in orbit computations are marked by colons.

As in Papers I-III, the best guess of the component's parameters ("models") is given in Table 5. The periods of outer sub-systems of the CPM type are estimated very roughly from their apparent separations. Each multiple system is discussed below individually.

\section{Discussion of individual systems}

ADS $61=$ HD 123. The components AB separated by 1".5 were mostly unresolved and show small velocity variations with a period of 47.5 days (Fig. 1). The corresponding orbit is given in Table 3. On a few occasions it was possible to measure the radial velocities of $\mathrm{A}$ and $\mathrm{B}$ separately, to establish that it is component B that is actually a spectroscopic binary. At this stage R. F. Griffin kindly communicated us the results of his study of ADS 61 (Griffin 1999) which agreed with our conclusions. His orbit is of better quality, but its independent confirmation from the RVM data seems to be worth publication, in view of the controversial results of Weber \& Strassmeier (1998). The value of $K_{1}$ is reduced by $\sim 3$ times because of dip blending; it is hence meaningless to compute the mass function from our orbit. For the same reason the orbital elements $e$ and $\omega$ differ from those found by Griffin.

ADS $2644=$ HD 22468. Slow variations of the radial velocity of B component are fitted to a 1152-day orbit of low quality (Fig. 2). The minimum secondary mass is $0.07 M_{\odot}$. Visual primary A is a 2.8-day double-lined chromospherically active binary V711 Tau with components of spectral types G5IV and K1IV. With the Hipparcos parallax, the components $\mathrm{Aa}, \mathrm{Ab}$ and $\mathrm{Ba}$ all lie some $1.2^{\mathrm{m}}$ above the Main Sequence (MS); Ba has a normal dip $E W$ and slow axial rotation. 

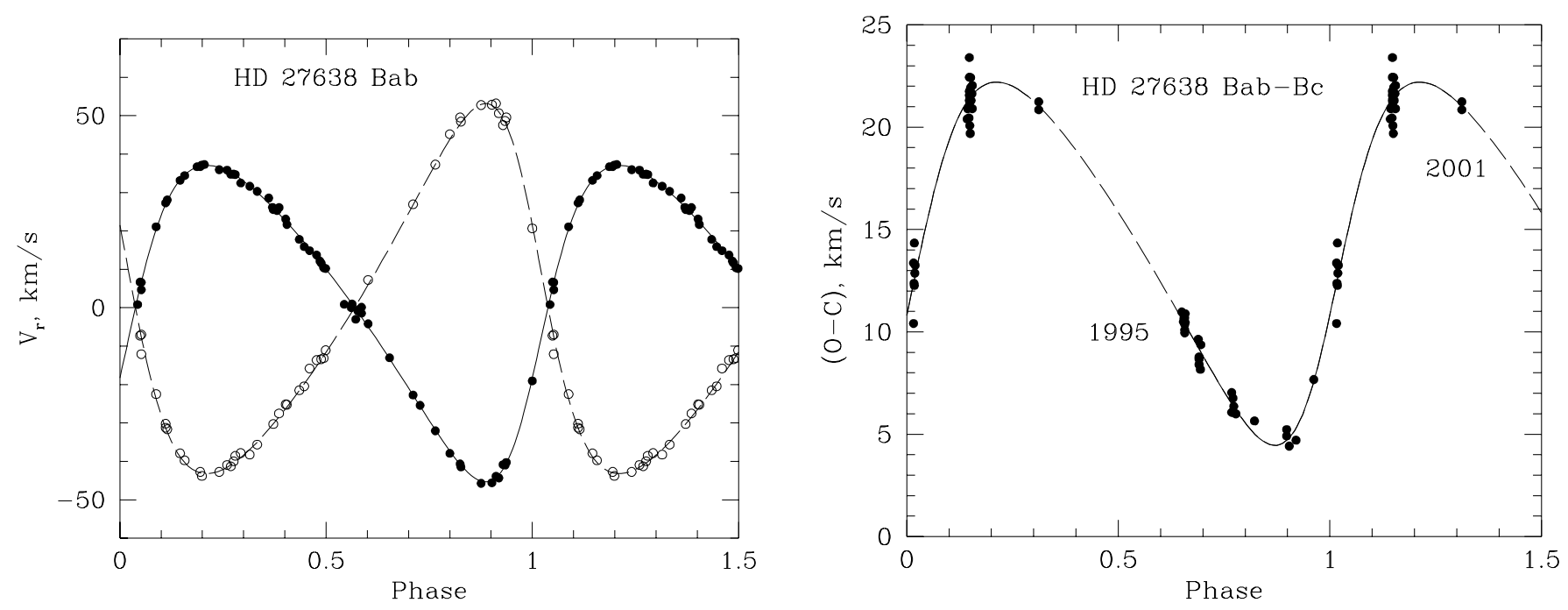

Fig. 3. The double-lined orbit of HD 27638Bab computed with removed long-period variations (left) and the residuals to this orbit fitted to a 8-yr orbit of Bab-Bc (right). Part of the orbit not yet covered by observations is plotted with a dashed line.
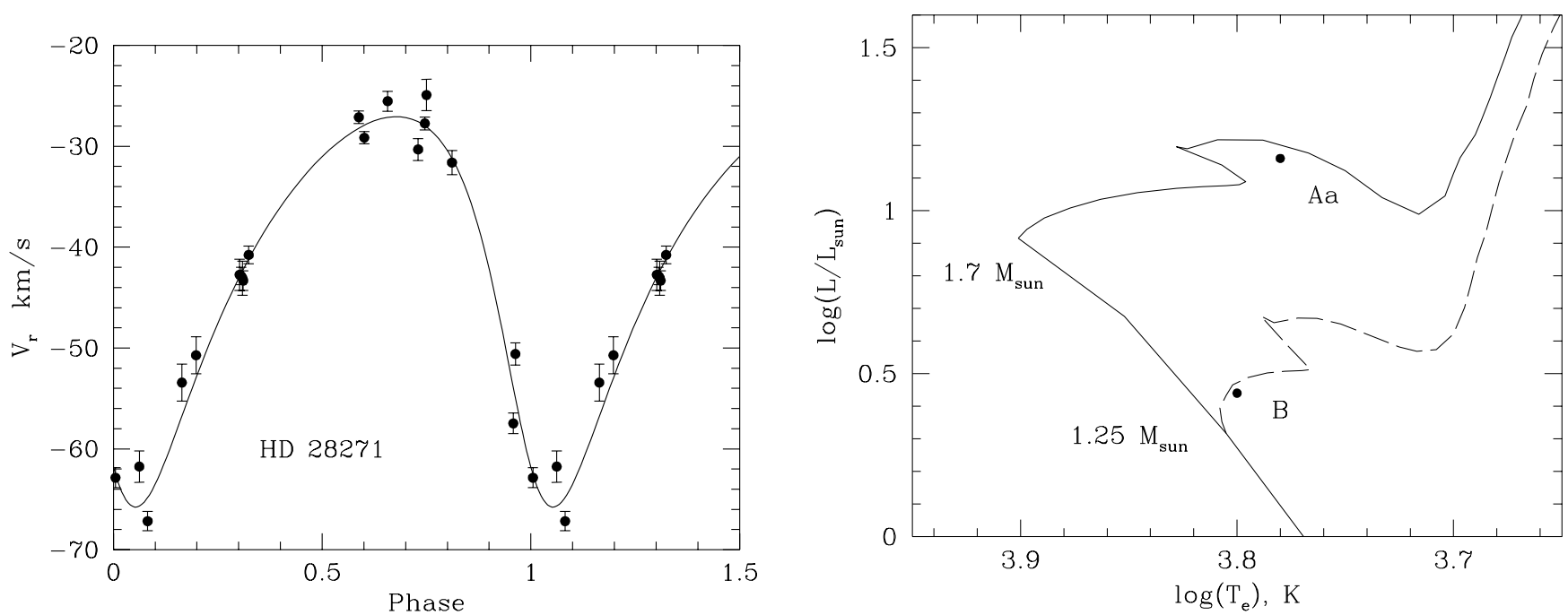

Fig. 4. Radial velocity curve of ADS $3243 \mathrm{~A}=$ HD 28271 (left). The evolutionary tracks for stars with masses of 1.12 and $1.7 M_{\odot}$ (Schaller et al. 1992), the Main Sequence and the positions of the components on the H-R diagram are shown on the right.

ADS $3161=$ HD 27638. The double-lined nature of the G2V secondary accompanying a young B-type primary $\chi$ Tau (estimated age $123 \mathrm{Myr}$, Lindroos 1986) was discovered by Martín et al. (1992), who also found a high lithium abundance. Independently, we discovered the double lines in 1995, after our first observations. However, in the next season, it became apparent that the systemic velocity changes revealed the existence of a third component, which here is called Bc. Only $\approx 70 \%$ of the long period is covered as yet, but a preliminary orbital solution is already reasonably well defined (Table 3 and Fig. 3 right). The discovery of this spectroscopic triple was announced in Tokovinin (1999b).

Elements of the short 17-day orbit of Bab (Fig. 3 left) were computed by subtracting the long-period motion from the data. Hence, the systemic velocity is zero by definition. Residuals from the Bab orbit are then used to refine the long orbit Bab-Bc (in fact, we used only good measurements with errors less than $0.5 \mathrm{kms}^{-1}$, and added two systemic velocities measured from blended dips in 2001). This iterative process was repeated twice. In Table 4 the final residuals to the short orbit (with longperiod motion removed) are given.

The mass ratio $\mathrm{Bb}: \mathrm{Ba}$ is 0.85 , the magnitude difference (as found from the equivalent width of the two dips) is $1.1^{\mathrm{m}}$. The system Bab can be modeled by two dwarfs of spectral types G0V and G6V (cf. Table 5). This model matches the observed $E W$, combined luminosity, combined $B-V$ color, magnitude difference and mass ratio, leaving little room for the third light. Both components $\mathrm{Ba}$ and $\mathrm{Bb}$ have slow axial rotation.

The nature of the long-period companion Bc remains a mystery: it is too massive! Even with the minimum mass of $\mathrm{Bab}$ of $1.13 M_{\odot}$, the minimum mass of $\mathrm{Bc}$ must be $0.9 M_{\odot}$; our model corresponds to the $\mathrm{Bc}$ minimum mass of $1.28 M_{\odot}$. Of course, the long orbit is still tentative, but 
its period cannot be much shorter, and the semi-amplitude is well defined, so the estimate of the Bc mass will not decrease when the definitive solution becomes available. We searched for the dip produced by Bc by taking long exposures at phases of maximum velocity difference in the Bab pair. Nothing was seen, which means that Bc must be at least 5 times fainter than $\mathrm{Bb}$ (dip contrast $<1 \%$ ).

The $\mathrm{Bc}$ component can be a close pair of $\mathrm{M}$ dwarfs with masses around $0.6 M_{\odot}$ each. Alternatively, it may be a single star obscured by circumstellar dust. The apparent semi-major axis of the Bab-c orbit must be $0{ }^{\prime \prime} 07$. In October 1997, when the B-component was observed by speckle interferometry in the $K$ band with the $6 \mathrm{~m}$ telescope (Balega et al. 2001), the estimated separation of the Bab-Bc pair was 0. '054 - just below the diffraction limit in the $K$ band, $0{ }^{\prime \prime} 09$.

ADS $3243=$ HD 28271. Both A and B components of this bright visual pair have correlation dips of very low contrast widened by a fast axial rotation. The scanning range of RVM is only $50 \mathrm{~km} \mathrm{~s}^{-1}$, and two overlapping scans were usually obtained to get the full dip profile. Radial velocities derived from such dips are of low accuracy, as seen in Fig. 4, and the dip parameters given in Table 2 are approximate.

The equivalent widths of the $\mathrm{A}$ and $\mathrm{B}$ dips correspond to their spectral type, F7, and to their $B-V$ colors. Yet, the minimum mass of $\mathrm{Ab}$ is $1.4 M_{\odot}$, i.e. comparable to the mass of Aa if it were an F7V dwarf. Looking at the photometric data for $\mathrm{A}$ and $\mathrm{B}$, it becomes apparent that although $\mathrm{A}$ is brighter than B by $1.8^{\mathrm{m}}$, it has a sightly redder color (the latter is confirmed by Shatskii 1998). Both components were measured by Hipparcos independently. Their concordant proper motions and radial velocities leave no doubt that it is a physical system. However, the Hipparcos parallax places A at $1^{\mathrm{m}}$ above the MS, and $\mathrm{B}$ at $1^{\mathrm{m}}$ below the MS.

We presume that the orbital motion of Aab with a period of $1.26 \mathrm{yr}$, unaccounted for in the reduction of Hipparcos data, caused a large error in the measured parallax. In fact, if $\mathrm{B}$ is indeed a normal F7V dwarf, the parallax must be around 12 mas. Then Aa must have an absolute magnitude of about $M_{V}=1.8^{\mathrm{m}}$. We place both components on the evolutionary tracks of Schaller et al. (1992) in Fig. 4 (right). It seems that the Aa component has a mass around $1.7 M_{\odot}$ and an age of $1.8 \mathrm{Gyr}$; it is leaving the Main Sequence. Its fast axial rotation explains a high chromospheric activity as manifested by a large X-ray flux of $10^{30} \mathrm{erg} \mathrm{s}^{-1}$ (Hünsch et al. 1998).

If this model is correct, the minimum mass of the invisible spectroscopic secondary $\mathrm{Ab}$ is $1.4 M_{\odot}$, and it could be either a slightly evolved F2V star or a close pair of low-mass dwarfs. The semi-major axis of Aab is 21 mas, which means that the photo-center must wobble with an amplitude of 10 mas. This triple system is an ideal target for future interferometric and astrometric work aimed at

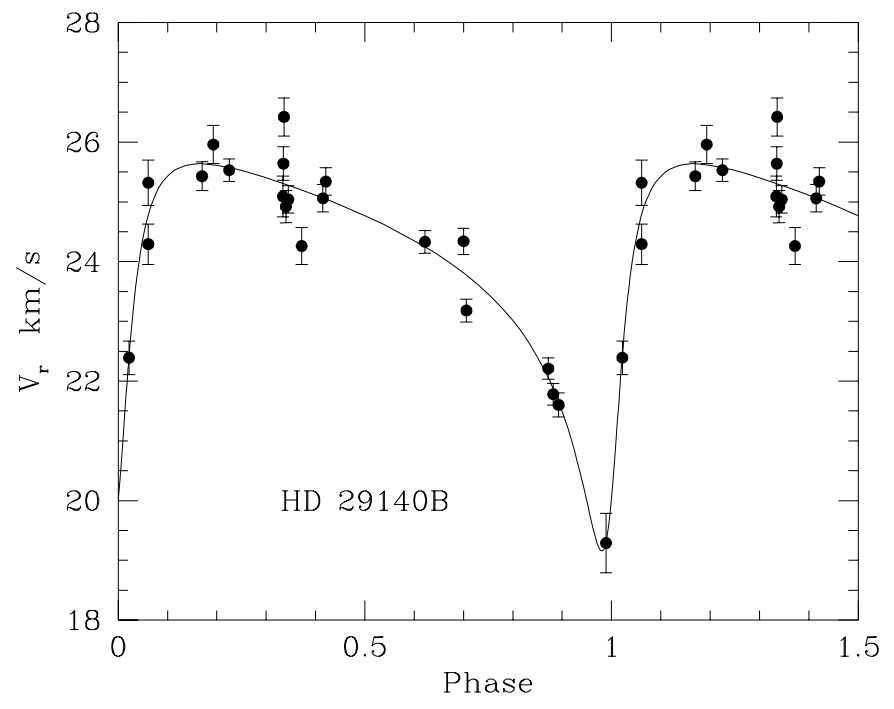

Fig. 5. Radial velocity curve of ADS 3317B = HD 29140B.

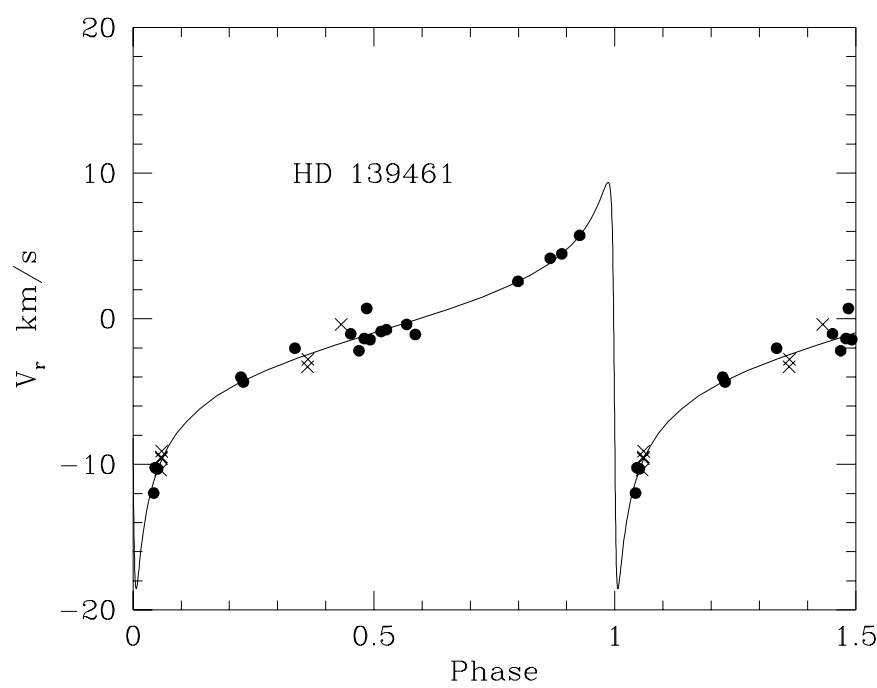

Fig. 6. Radial velocity curve for a highly eccentric orbit of ADS $9728 \mathrm{~A}=$ HD 139461. The measurements by Struve \& Zebergs (1959) are marked by crosses.

determining the physical parameters of the components which happen to transit a rare evolutionary stage.

ADS $3317=$ HD 29140. The visual primary $A$ is a quadruple system, consisting of two close spectroscopic pairs forming a 18-yr interferometric binary (Balega et al. 1999). The parameters of those 4 stars as listed in Table 5 are not yet well established. Here we show that B is also a binary with a low-mass companion on a 3.7-yr orbit (Fig. 5). Thus the whole system is sextuple. The semimajor axis of the Bab orbit must be 6 mas or more. The orbital motion may be detectable in the future by precise astrometry in order to find the inclination and the mass of $\mathrm{Bb}$.

ADS $9728=$ HD 139461/60. The bright pair ADS 9728 with negative declination could be observed only 
Table 2. Basic observational data.

\begin{tabular}{|c|c|c|c|c|c|c|c|c|c|c|c|}
\hline ADS & Comp. & $\begin{array}{l}\text { Sp. } \\
\text { type }\end{array}$ & $V$ & $B-V$ & $\begin{array}{c}\mu_{\alpha} \\
\operatorname{mas} / y\end{array}$ & $\begin{array}{c}\mu_{\delta} \\
\mathrm{mas} / \mathrm{y}\end{array}$ & $\begin{array}{c}\pi \\
\text { mas }\end{array}$ & $\begin{array}{c}V_{\mathrm{r}} \\
\mathrm{km} \mathrm{s}^{-1}\end{array}$ & $N_{\text {obs }}$ & $\begin{array}{c}E W \\
\mathrm{~km} \mathrm{~s}^{-1}\end{array}$ & $\begin{array}{r}V \sin i \\
\mathrm{~km} \mathrm{~s}^{-1}\end{array}$ \\
\hline 61 & $\begin{array}{l}\mathrm{A} \\
\mathrm{B}\end{array}$ & $\begin{array}{l}\text { G5V } \\
\text { G8V }\end{array}$ & $\begin{array}{l}6.34 \\
7.27\end{array}$ & $\begin{array}{c}0.67 \\
-\end{array}$ & $\begin{array}{l}247 \\
280\end{array}$ & $\begin{array}{l}18 \\
56\end{array}$ & $49.3 \pm 1.0$ & $-13.8 \pm 0.2$ & 24 & $2.71 \pm 0.02$ & - \\
\hline 2644 & $\begin{array}{l}\mathrm{A} \\
\mathrm{B}\end{array}$ & $\begin{array}{l}\text { G9V } \\
\text { K6V }\end{array}$ & $\begin{array}{l}5.91 \\
8.79\end{array}$ & $\begin{array}{l}0.86 \\
1 / 03\end{array}$ & -33 & -163 & $34.5 \pm 0.9$ & $\begin{array}{c}-15.0 \\
-15.9 \pm 0.2\end{array}$ & $\begin{array}{c}- \\
12\end{array}$ & $\begin{array}{c}- \\
2.85 \pm 0.09\end{array}$ & $\begin{array}{c}- \\
<4.7\end{array}$ \\
\hline 3161 & $\begin{array}{l}\mathrm{A} \\
\mathrm{B}\end{array}$ & $\begin{array}{l}\text { B9V } \\
\text { G2V }\end{array}$ & $\begin{array}{l}5.37 \\
8.41\end{array}$ & $\begin{array}{c}-0.05 \\
0.58\end{array}$ & $\begin{array}{c}20 \\
(38)\end{array}$ & $\begin{array}{l}-18 \\
(-12)\end{array}$ & $\begin{array}{c}12.2 \pm 1.0 \\
-\end{array}$ & $\begin{array}{c}17.0 \\
14.0 \pm 0.3\end{array}$ & $\begin{array}{c}- \\
90\end{array}$ & $\begin{array}{c}- \\
1.51 \pm 0.02 \\
0.75 \pm 0.02\end{array}$ & $\begin{array}{c}- \\
2.9 \pm 0.6 \\
0\end{array}$ \\
\hline 3243 & $\begin{array}{l}\mathrm{A} \\
\mathrm{B}\end{array}$ & F7V & $\begin{array}{l}6.38 \\
8.17\end{array}$ & $\begin{array}{l}0.54 \\
0.48\end{array}$ & $\begin{array}{l}23 \\
14\end{array}$ & $\begin{array}{l}-20 \\
-22 \\
\end{array}$ & $\begin{array}{l}18.4 \pm 1.6 \\
21.5 \pm 7.5 \\
\end{array}$ & $\begin{array}{l}-41.6 \pm 0.6 \\
-43.3 \pm 0.4 \\
\end{array}$ & $\begin{array}{c}20 \\
9 \\
\end{array}$ & $\begin{array}{l}2.05 \pm 0.07 \\
2.10 \pm 0.15 \\
\end{array}$ & $\begin{array}{l}33: \\
22:\end{array}$ \\
\hline 3317 & $\begin{array}{l}\mathrm{A} \\
\mathrm{B}\end{array}$ & $\begin{array}{l}\text { A5m } \\
\text { F8V }\end{array}$ & $\begin{array}{l}4.25 \\
7.84\end{array}$ & $\begin{array}{l}0.18 \\
0.54\end{array}$ & $\begin{array}{c}45 \\
(38)\end{array}$ & $\begin{array}{l}-52 \\
(-50)\end{array}$ & $21.7 \pm 0.8$ & $\begin{array}{c}29.0 \\
24.0 \pm 0.2\end{array}$ & 21 & $2.26 \pm 0.03$ & $4.9 \pm 0.4$ \\
\hline 9728 & $\begin{array}{l}\mathrm{A} \\
\mathrm{B}\end{array}$ & $\begin{array}{l}\text { F6V } \\
\text { F6IV }\end{array}$ & $\begin{array}{l}6.49 \\
6.56\end{array}$ & $\begin{array}{l}0.49 \\
0.52\end{array}$ & $\begin{array}{c}17 \\
1\end{array}$ & $\begin{array}{l}-27 \\
-24\end{array}$ & $\begin{array}{l}40.2 \pm 3.6 \\
44.2 \pm 4.7\end{array}$ & $\begin{array}{c}-1.3 \pm 0.2 \\
1.2 \pm 0.1\end{array}$ & $\begin{array}{l}26 \\
18\end{array}$ & $\begin{array}{l}1.83 \pm 0.03 \\
1.85 \pm 0.03\end{array}$ & $\begin{array}{l}5.3 \pm 0.4 \\
6.8 \pm 0.4\end{array}$ \\
\hline 11328 & $\begin{array}{l}\mathrm{A} \\
\mathrm{B} \\
\mathrm{C}\end{array}$ & G5 & $\begin{array}{c}9.01 \\
9.15 \\
10.36\end{array}$ & $\begin{array}{l}0.61 \\
0.64 \\
0.62\end{array}$ & 47 & 132 & $15.7 \pm 1.7$ & $\begin{array}{c}-35.9 \pm 0.4 \\
-31.7 \pm 0.4 \\
-21.5:\end{array}$ & $\begin{array}{l}22 \\
23 \\
15\end{array}$ & $\begin{array}{c}3.02: \\
2.93: \\
2.10 \pm 0.11\end{array}$ & $\begin{array}{l}- \\
- \\
-\end{array}$ \\
\hline 12145 & $\begin{array}{l}\mathrm{B} \\
\mathrm{A} \\
\mathrm{C}\end{array}$ & $\begin{array}{l}\text { G6V } \\
\text { G9V }\end{array}$ & $\begin{array}{l}8.15 \\
8.60 \\
9.10\end{array}$ & $\begin{array}{c}0.73 \\
0.79 ?\end{array}$ & $\begin{array}{l}-228 \\
-238 \\
-246\end{array}$ & $\begin{array}{l}-106 \\
-99 \\
-84\end{array}$ & $20.5 \pm 1.3$ & $\begin{array}{l}25.6 \pm 0.1 \\
26.1 \pm 0.1 \\
22.8 \pm 0.6\end{array}$ & $\begin{array}{l}35 \\
32 \\
32\end{array}$ & $\begin{array}{c}2.53 \pm 0.03 \\
1.68: \\
1.00:\end{array}$ & $\begin{array}{c}<2.7 \\
- \\
-\end{array}$ \\
\hline
\end{tabular}

Table 3. Elements of spectroscopic orbits.

\begin{tabular}{llllllllll}
\hline \hline ADS & $P$ & $T$ & $e$ & $\omega$ & $\begin{array}{l}K_{1} \\
\mathrm{~km} \mathrm{~s}^{-1}\end{array}$ & $\begin{array}{l}K_{2} \\
\mathrm{~km} \mathrm{~s}^{-1}\end{array}$ & $\begin{array}{l}\gamma \\
\mathrm{km} \mathrm{s}^{-1}\end{array}$ & $\begin{array}{l}\sigma_{V} \\
f(m) \text { or } M \sin ^{3} i \\
M_{\odot}\end{array}$ \\
\hline 61 AB & 47.51 & 50798.52 & 0.418 & 289 & 2.86 & - & -13.79 & 24 & - \\
123 & \pm 0.04 & \pm 0.95 & \pm 0.056 & \pm 11 & \pm 0.21 & & \pm 0.14 & 0.51 & \\
2644 B & 1152 & 50362 & 0.40 & 88 & 1.76 & - & -15.94 & 12 & 0.0005 \\
22468 B & \pm 44 & \pm 85 & \pm 0.22 & \pm 31 & \pm 0.55 & & \pm 0.17 & 0.33 & \pm 0.0004 \\
3161 Bab & 17.60249 & 50000.617 & 0.297 & 249.9 & 41.19 & 48.18 & 0 & 105 & 0.610 \\
27638 Bab & \pm 0.00028 & \pm 0.029 & \pm 0.003 & \pm 0.6 & \pm 0.16 & \pm 0.24 & $*$ & $0.90 / 1.21$ & \pm 0.006 \\
3161 Bab-c & 2951 & 51010 & 0.262 & 254 & 8.87 & - & 14.06 & 57 & 0.192 \\
27638 Bab-c & \pm 194 & \pm 32 & \pm 0.051 & \pm 6 & \pm 0.18 & & \pm 0.28 & 0.69 & \pm 0.014 \\
3243 A & 460.7 & 50456 & 0.314 & 143 & 19.35 & - & -41.58 & 19 & 0.295 \\
28271 A & \pm 3.5 & \pm 8 & \pm 0.048 & \pm 7 & \pm 0.88 & & \pm 0.59 & 3.06 & \pm 0.037 \\
3317 B & 1350 & 50498 & 0.663 & 223 & 3.24 & - & 23.97 & 21 & 0.0020 \\
29140 B & \pm 35 & \pm 34 & \pm 0.075 & \pm 9 & \pm 0.44 & & \pm 0.18 & 0.41 & \pm 0.0005 \\
9728 A & 887.66 & 51016.8 & 0.900 & 105 & 13.96 & - & -1.33 & 26 & $0.019:$ \\
139461 & \pm 0.21 & \pm 3.9 & $*$ & $*$ & \pm 0.43 & & & 0.44 & 0.067 \\
11328 B & 126.38 & 50498.6 & 0.331 & 319.0 & 18.27 & - & -31.68 & 23 & \pm 0.005 \\
$169816 \mathrm{~B}$ & \pm 0.22 & \pm 2.1 & \pm 0.036 & \pm 6.0 & \pm 0.44 & & \pm 0.37 & 1.01 & 0.126 \\
12145 C & 522.7 & 50761.0 & 0.606 & 58.3 & 16.66 & - & 22.83 & 33 & \pm 0.012 \\
$179484 \mathrm{C}$ & \pm 2.2 & \pm 2.9 & \pm 0.041 & \pm 3.6 & \pm 1.09 & & \pm 0.29 & 0.92 & \\
\hline \hline
\end{tabular}

during a limited period each year. The primary component HD 139461 was discovered as a spectroscopic binary by Christie \& Wilson (1938) and confirmed by Struve \& Zebergs (1959), although neither of them attempted to determine an orbit. The orbital period is now known to be $2.4 \mathrm{yr}$, but it is evident that the eccentricity is high and not yet constrained by the data (Fig. 6). The data from Struve \& Zebergs were used to refine the period, after a correction of $-1.8 \mathrm{~km} \mathrm{~s}^{-1}$ that brings the mean velocity of the B component into agreement with our measurements. Elements $e$ and $\omega$ are kept fixed in our preliminary orbital solution.

A special observing campaign must be planned to "catch" the periastron passage which may be very short (e.g. 2 days in case of ADS 11061A, Tokovinin 1995). Unfortunately, the last periastron passage occurred in January 2001 when the object was difficult to reach even from the southern hemisphere. This means that there are 
Table 5. Models of multiple systems.

\begin{tabular}{|c|c|c|c|c|c|c|c|c|c|}
\hline $\begin{array}{l}\mathrm{ADS} \\
\pi, \text { mas } \\
m-M\end{array}$ & Comp. & $V$ & $B-V$ & $\begin{array}{l}\text { Sp. } \\
\text { type }\end{array}$ & $\begin{array}{l}\text { Mass, } \\
M_{\odot}\end{array}$ & Sys. & Type & Period & $\begin{array}{l}\text { Sep. } \\
\text { S }\end{array}$ \\
\hline 61 & $\mathrm{~A}$ & 6.34 & & G3V & 0.98 & $\mathrm{AB}$ & $\mathrm{V}$ & $106.8 \mathrm{y}$ & 1.43 \\
\hline 49.3 & $\mathrm{Ba}$ & 7.99 & & G9V & 0.86 & Bab & SB1 & $47.7 \mathrm{~d}$ & 0.013 \\
\hline 1.5 & $\mathrm{Bb}$ & 10.4 & & M3V & 0.31 & & & & \\
\hline 2644 & $\mathrm{Aa}$ & 6.24 & & G5IV & 0.90 & $\mathrm{AB}$ & $\mathrm{V}$ & $2100 \mathrm{y}$ & 8.02 \\
\hline 34.5 & $\mathrm{Ab}$ & 7.24 & & K1IV & 0.76 & Aab & SB2 & $2.84 \mathrm{~d}$ & 0.0016 \\
\hline 1.2 & $\begin{array}{l}\mathrm{Ba} \\
\mathrm{Bb}\end{array}$ & 8.83 & 0.99 & $\begin{array}{l}\mathrm{K} 6 \mathrm{~V} \\
\mathrm{M}\end{array}$ & $\begin{array}{l}0.65 \\
>0.07\end{array}$ & $\mathrm{Bab}$ & SB1 & $1152 \mathrm{~d}$ & 0.066 \\
\hline 3161 & $\mathrm{~A}$ & 5.37 & -0.05 & B9V & 3.80 & $\mathrm{AB}$ & CPM & $21000 \mathrm{y}$ & 19.4 \\
\hline 12.2 & $\mathrm{Ba}$ & 8.75 & 0.54 & GOV & 1.10 & $\mathrm{Bac}$ & SB1 & $8.1 \mathrm{y}$ & 0.073 \\
\hline 4.6 & $\begin{array}{l}\mathrm{Bb} \\
\mathrm{Bc}\end{array}$ & $\begin{array}{l}9.83 \\
>12\end{array}$ & 0.69 & $\begin{array}{l}\text { G6V } \\
?\end{array}$ & $\begin{array}{l}0.94 \\
>1.2\end{array}$ & $\mathrm{Bab}$ & SB2 & $17.6 \mathrm{~d}$ & 0.002 \\
\hline 3243 & $\mathrm{Aa}$ & 6.39 & 0.52 & F7III & 1.7 & $\overline{\mathrm{AB}}$ & CPM & $20000 y$ & 14.2 \\
\hline 12 & $\mathrm{Ab}$ & & & F2V? & $>1.4$ & Aab & SB1 & $461 \mathrm{~d}$ & 0.020 \\
\hline 4.6 & B & 8.31 & 0.48 & F7V & 1.2 & & & & \\
\hline 3317 & $\mathrm{Aa}$ & 4.52 & & $\mathrm{~A} 2 \mathrm{Vm}$ & 1.9 & $\mathrm{AB}$ & CPM & $70000 \mathrm{y}$ & 69.7 \\
\hline 21.7 & $\mathrm{Ab}$ & 6.92 & & F7V? & 1.12 & $\mathrm{AP}$ & $\mathrm{V}$ & $18.05 \mathrm{y}$ & 0.241 \\
\hline \multirow[t]{4}{*}{3.3} & $\mathrm{~Pa}$ & 7.16 & & F7V? & 1.12 & Aab & SB2 & $3.57 \mathrm{~d}$ & 0.0014 \\
\hline & $\mathrm{Pb}$ & 7.16 & & F7V? & 1.12 & $\mathrm{Pab}$ & SB? & - & - \\
\hline & $\mathrm{Ba}$ & 7.84 & 0.54 & F8V & 1.2 & $\mathrm{Bab}$ & SB1 & $1350 \mathrm{~d}$ & 0.057 \\
\hline & $\mathrm{Bb}$ & & & $\mathrm{M} ?$ & $>0.15$ & & & & \\
\hline 9728 & $\mathrm{Aa}$ & 6.48 & 0.50 & F6V & 1.25 & $\overline{\mathrm{AB}}$ & CPM & $5000 \mathrm{y}$ & 11.8 \\
\hline 27 & $\mathrm{Ab}$ & & & $\mathrm{M} ?$ & $>0.36$ & Aab & SB1 & $888 \mathrm{~d}$ & 0.059 \\
\hline 2.8 & B & 6.50 & 0.52 & F6V & 1.25 & & & & \\
\hline 11328 & $\mathrm{~A}$ & 9.08 & 0.61 & G5V & 0.94 & $\overline{\mathrm{AB}}$ & $\mathrm{v}$ & $1400 \mathrm{y}$ & 2.60 \\
\hline 15.7 & $\mathrm{Ba}$ & 9.22 & 0.64 & G5V & 0.94 & Bab & SB1 & $126 \mathrm{~d}$ & 0.009 \\
\hline 1.5 & $\mathrm{Bb}$ & & & K-M & $>0.5$ & & & & \\
\hline 12145 & $\mathrm{Ba}$ & 8.15 & 0.73 & G6V & 0.90 & $\overline{\mathrm{BA}}$ & CPM & $2000 y$ & 4.50 \\
\hline 20.5 & $\mathrm{Bb}$ & $?$ & & $?$ & $?$ & Bab & SB1 & $>9 y$ & $>0.1$ \\
\hline \multirow[t]{3}{*}{3.4} & $\mathrm{~A}$ & 8.60 & 0.77 & G7V & 0.85 & $\mathrm{AC}$ & $\mathrm{V}$ & $63 \mathrm{y}$ & 0.37 \\
\hline & $\mathrm{Ca}$ & 9.10 & 0.83 & G9V & 0.80 & $\mathrm{Cab}$ & SB1 & $523 \mathrm{~d}$ & 0.029 \\
\hline & $\mathrm{Cb}$ & $12 ?$ & & K8V? & $>0.64$ & & & & \\
\hline
\end{tabular}

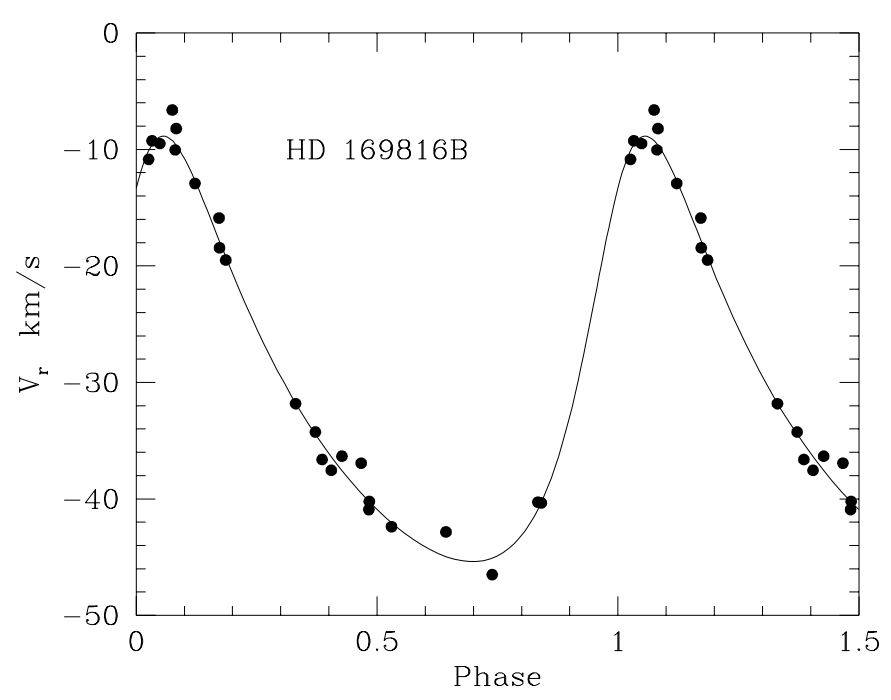

Fig. 7. Radial velocity curve of ADS 11328B = HD 169816B.

at least 3 years to wait for a definitive orbit, which justifies the publication of the preliminary solution.
The model of the system presented in Table 5 assumes that both components have normal luminosities, and that the Hipparcos parallax is erroneous because of the orbital motion of A (its large error of 4 mas can be viewed as indirect evidence of the astrometric perturbation in the parallax solution).

ADS $11328=$ HD 169816. The observations of AB with a separation of only 2.'6 were always difficult. Components could be measured separately only under good seeing, without guaranteeing that the resolution was indeed complete. Nevertheless, the orbit presented in Fig. 7 almost qualifies as a definitive one. When the velocities of $\mathrm{A}$ and $\mathrm{B}$ differed by more than $15 \mathrm{~km} \mathrm{~s}^{-1}$ they could also be measured as an unresolved double-lined system. The profile parameters in Table 2 are unreliable because of components blending at the slit.

The third visual component ADS $11328 \mathrm{C}$ was observed and found to be a double-lined binary. Most of the velocities given in Table 4 are obtained from the unresolved dip of varying contrast, they concentrate around $-20 \mathrm{~km} \mathrm{~s}^{-1}$ 

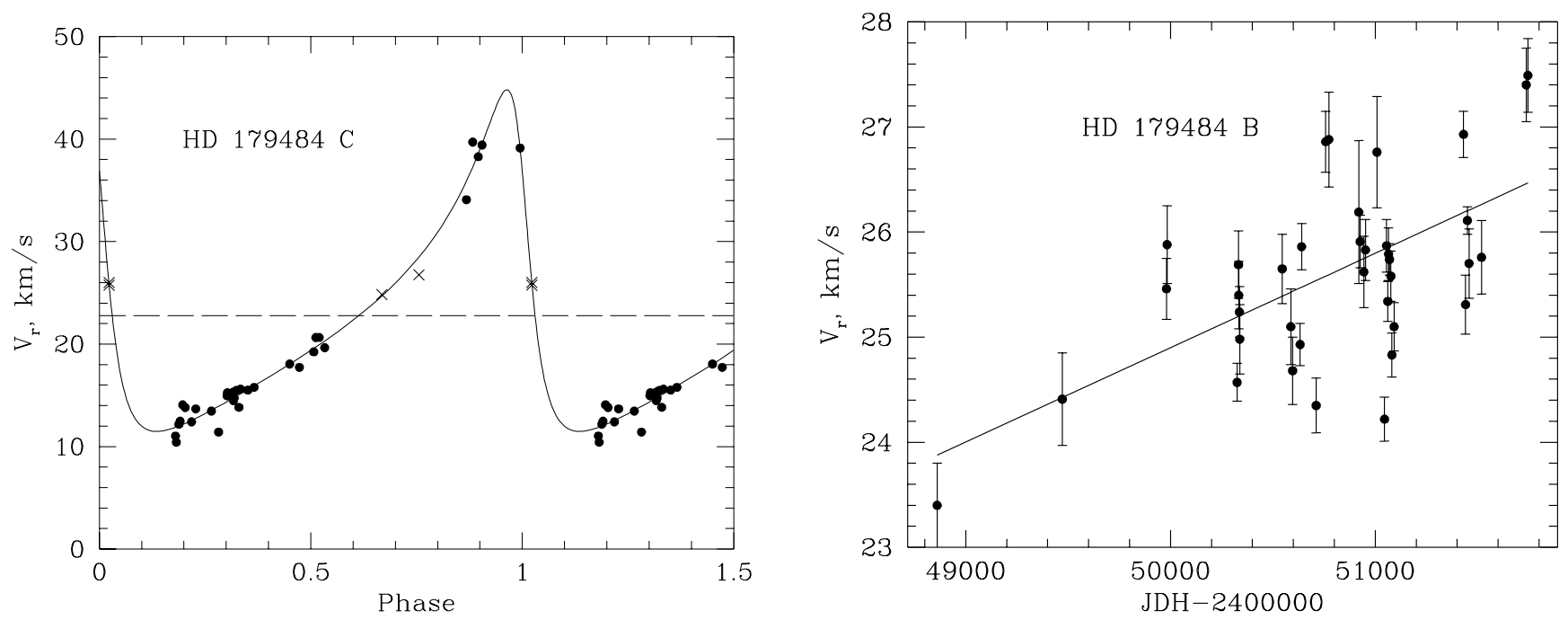

Fig. 8. Radial velocity curve of ADS $12145 \mathrm{C}$ (left) and the radial velocities of B as a function of Julian date with a linear trend fitted (right).

indicating that it is an optical companion. Dip contrast is smaller than that of $\mathrm{A}$ and $\mathrm{B}$, also suggesting that $\mathrm{C}$ is in fact a background star in this rich galactic-plane field. For this reason we stopped the observations of $\mathrm{C}$ and did not attempt to derive its orbit. The spectral type of $\mathrm{C}$ can be estimated as G3V from the $B-V$ color or as $\mathrm{F} 7 \mathrm{~V}$ from the $\operatorname{dip} E W$.

ADS $12145=$ HD 179484. This star was recognized as double for the first time by Struve in 1830 and was named $\Sigma 2481$. Both components are nearly equal, but the northern one is slightly brighter, and hence was called A. Since its discovery, the pair $\mathrm{AB}$ has opened up from $3 . ! 8$ to 4.' 6 and moved clockwise in position angle from 234 to 204 degrees.

Later, in 1856, Secchi (1860) found that one of the visual components is a closer binary SE 2. In the ADS (Aitken 1932) this close pair is attributed to the component $\mathrm{B}$ and is called BC. The WDS (Worley \& Douglass 1997) continues this notation, but, nevertheless, in the Notes it is said that "SE 2 is the NP component of STF 2481 (van den Bos)". Heintz (1991) confirms that the close pair is indeed the northern component of $\Sigma 2481$, the same follows from the Double and Multiple Star Annex to the Hipparcos catalogue (ESA 1997). We keep this notation of the components: the close pair is hence called AC. Although the northern system AC is brighter than B in combined light, the component $\mathrm{B}$ is brighter than either A or $\mathrm{C}$ taken separately.

We measured the radial velocities of ADS 12145 from 1994 to 2000 . The relatively small separation between AC and $\mathrm{B}$ made it necessary to rotate the RVM instrument in order to turn the slit in the unusual east-west direction for better discrimination between the components. Even with this precaution, the separation of the light from AC and $\mathrm{B}$ was not complete under bad seeing conditions.
It was found that the radial velocity of $\mathrm{B}$ is apparently constant, while that of $\mathrm{AC}$ changes by few $\mathrm{km} \mathrm{s}^{-1}$. Those variations were accompanied by the changing width and contrast of the correlation dip. Here we deal with a triple system: the strongest dip of A is stationary, and the dip of $\mathrm{C}$ changes its position, although these two dips always remain blended.

After several trials, we found a period of 520 days and selected the dip parameters (contrasts of $6 \%$ for $\mathrm{C}$ and $10 \%$ for A, a $F W H M$ of $15 \mathrm{~km} \mathrm{~s}^{-1}$ for both) that lead to an acceptable orbital solution for the Cab motion (Fig. 8 left) when the individual velocities were extracted from the blended dips. The elements of the final spectroscopic orbit of $\mathrm{C}$ computed with the adopted dip parameters are given in Table 3. Four unresolved measurements of $\mathrm{AC}$ corresponding to the superposition of their dips were also used in orbit computation with reduced weight; these points are shown by crosses.

The radial velocities of the constant dip (component A), when fitted to this 522-day orbit, show only a slight trace of the orbital motion (formally, $K_{1}=-0.96 \mathrm{~km} \mathrm{~s}^{-1}$, $\left.V_{0}=26.14 \pm 0.20 \mathrm{kms}^{-1}\right)$. This proves that the dip parameters adopted in de-blending are almost correct. No additional periods were found in the velocities of A. Note that the average velocity of $\mathrm{A}$ is different from that of $\mathrm{C}$ because of the AC motion in its 63-yr visual orbit. The components AC are actually close to apastron, hence the variation of the velocity caused by the visual orbit during the time of our observations can be neglected.

Taking the mass of $\mathrm{Ca}$ to be $0.8 M_{\odot}$ according to its spectral type, we obtain from the orbit the minimum mass of the invisible component $\mathrm{Cb}, 0.64 M_{\odot}$. This corresponds to a dwarf star of the late $\mathrm{K}$ spectral type which must have an apparent magnitude around $12^{\mathrm{m}}$. The contrast of its dip would be $0.4 \%$, i.e. not noticeable. Alternatively, $\mathrm{Cb}$ can be a white dwarf. We note that the mass ratio of the AC system that follows from the adopted masses 
(Table 5), $\beta=M_{\mathrm{C}} / M_{\mathrm{A}}=0.64$, does not agree with the astrometric determination of Heintz (1991) who finds $\beta=0.454$. The amplitude of the wobble in the AC orbital motion that is caused by the Cab sub-system is estimated to be 0. . 01 ; it can be detectable by speckle interferometry.

Turning to the radial velocities of B (Fig. 8 right), we detect an apparent trend of $0.3 \mathrm{~km} \mathrm{~s}^{-1}$ per year. The measurement obtained in 1992 by Smekhov \& Tokovinin (1993) is added to this plot (originally it was attributed to the wrongly identified southern component BC). Thus, B itself is a spectroscopic binary with a period longer than 9 yr and, apparently, a low-mass secondary which does not manifest by changing the dip shape. The total number of components known to date in ADS 12145 is thus five.

\section{Conclusions}

Among the nine spectroscopic sub-systems presented in this paper, only three were known previously and rediscovered by us independently. Compared to the previous papers of this series, the objects studied here are more difficult, but also more interesting. They include a young spectroscopic triple HD 27638B with an unseen and massive tertiary, an interesting evolutionary stage of HD 28271A, an additional sub-system in the sextuple stellar system $\chi$ Tau, a candidate high-eccentricity binary HD 139461, and two additional components in the visual triple ADS 12145. In these cases further studies with complementary techniques (interferometry, precise astrometry) will be needed.

Acknowledgements. The authors are grateful to the personnel of the Simeis observatory for the possibility to use the $1 \mathrm{~m}$ telescope and to M. Mayor who made available CORAVEL in 1994. Some measurements were secured by our colleagues N. Samus, M. Smekhov, E. Glushkova, M. Sachkov, A. Rastorgouev. We thank B. Mason for retrieving the data on ADS 12145 from the Washington database. SIMBAD operated by the University of
Strasbourg was consulted. This work was partially supported by the grant from the Russian State Committee of Science and Higher Education and by the grant MPB000 from the International Science Foundation.

\section{References}

Aitken, R. G. 1932, New General Catalogue of Double Stars, Carnegie Inst., No. 417

Balega, I. I., Balega, Yu. Yu., Hofmann, K.-H., et al. 1999, PAZh, 25, 910 (AstL, 25, 797)

Balega, I. I., Balega, Y. Y., Hofmann, K.-H., et al. 2001, PAZh, submitted

Baranne, A., Mayor, M., \& Poncet, J. L. 1979, Vistas Astron., 23, 279

Christie, W. H., \& Wilson, O. C. 1938, ApJ, 88, 34

ESA, 1997, European Space Agency, SP-1200

Griffin, R. F. 1999, Observatory, 119, 27

Heintz, W. 1991, AJ, 101, 1071

Hünsch, M., Schmitt, J. H. M. M., \& Voges W. 1998, A\&AS, 132,155

Martín, E. L., Magazzu, A., \& Rebolo, R. 1992, A\&A, 257, 186

Lindroos, K. P. 1986, A\&A, 156, 223

Schaller, G., Schaerer, D., Meynet, G., \& Maeder A. 1992, A\&AS, 96, 269

Secchi, A. 1860, Cat. Stellie Doppie Col. Rome

Shatskii, N. I. 1998, PAZh, 24, 307

Smekhov, M. G., \& Tokovinin, A. A. 1993, PAZh, 19, 193

Struve, O., \& Zebergs, V. 1959, AJ, 64, 219

Tokovinin, A. A. 1987, AZh, 64, 196 (SvA, 31, 98)

Tokovinin, A. A. 1990, PAZh, 16, 52 (SvAL, 16, 24)

Tokovinin, A. A. 1995, PAZh, 21, 286

Tokovinin, A. A. 1997a, A\&AS, 121, 71, Paper I

Tokovinin, A. A. 1997b, A\&AS, 124, 75

Tokovinin, A. A. 1998, PAZh, 24, 343, Paper II

Tokovinin, A. A. 1999a, A\&AS, 136, 373, Paper III

Tokovinin, A. A. 1999b, ASP Conf. Ser., 185, 347

Weber, M., \& Strassmeier, K. G. 1998, IBVS, 4573, 1

Worley, C. E., \& Douglass, G. G. 1997, A\&AS, 125, 523 